\title{
CYCLOTOMIC SPLITTING FIELDS FOR GROUP CHARACTERS
}

\author{
MARK BENARD 1
}

\begin{abstract}
This paper is concerned with cyclotomic splitting fields for a real-valued irreducible character of a finite group. The fields considered are of the form $Q\left(\epsilon_{m}\right)$, where $m$ is either an odd prime or a power of 2 .
\end{abstract}

Let $\chi$ be an irreducible character of $G$ and let $\epsilon_{m}$ be a primitive $m$ th root of unity. A famous theorem of Richard Brauer states that if $m$ is the exponent of $G$, then $Q\left(\epsilon_{m}\right)$ is a splitting field for $G$. In a paper where he gives his second proof of this theorem, Brauer states the following proposition without proof [2, Theorem 3]: If $\chi$ is a real-valued character of $G$, then there exists an element of $G$ whose order $m$ is either an odd prime or a power of 2 such that $Q\left(\epsilon_{m}\right)$ splits $\chi$. The examples given below show that this proposition is actually false. One weaker theorem is proved by B. Fein [3]. The Theorem given below is another attempt to substitute for Brauer's proposition.

Let $k$ be a field of characteristic 0 . The pair $(G, \chi)$ is said to be $k$-special if there exists a normal, cyclic, self-centralizing subgroup $A$ of $G$ and a faithful linear character $\lambda$ of $A$ such that $\chi=\lambda^{G}$ and $G / A$ acts on $\lambda$ as $\mathrm{Gal}(k(\lambda) / k(\chi))$. Many questions on the Schur index reduce to considering such $k$-special pairs. Basic results on the Schur index can be found in Yamada [4].

Theorem. Suppose that $\chi$ is a real-valued character of $G$ and $G$ contains no elements of order $4 n$ with $n$ odd and $n>1$. Then there exists an integer $m$ dividing the exponent of $G$ such that $m$ is either an odd prime or 4, and such that $Q\left(\epsilon_{m}\right)$ splits $\chi$.

Proof. To prove the Theorem, it is necessary to show that the Schur index $m_{F}(\chi)$ equals 1 for some field $F=Q\left(\epsilon_{m}\right)$ as specified above. Since $\chi$ is real-valued, then $m_{Q}(\chi) \leq 2$ by the Brauer-Speiser theorem. By the the Brauer-Witt theorem, it suffices to consider $Q(\chi)$-special pairs $(G, \chi)$ where $G / A$ is a 2-group.

If $G$ is a 2-group, then $m_{Q}(\chi)=1$ if $\exp (G)=2$. If $4 \mid \exp (G)$, then $m$

Received by the editors October 25, 1974.

AMS (MOS) subject classifications (1970). Primary 20C15.

Key words and phrases. Schur index, Brauer-Speiser theorem, Brauer-Witt theorem.

${ }^{1}$ This research was supported by NSF Grant GP-29437.

Copyright $\odot 1975$, American Mathematical Society 
$=4$ satisfies the conclusion of the Theorem. For the remainder of the proof, assume that there exists an odd prime $q$ which divides $|G|$. It will be shown that $F=Q\left(\epsilon_{q}\right)$ splits $\chi$.

Assume $m_{Q}(X)=2$. Let $p$ be a prime such that $m_{Q_{p}}(\chi)=2$. Let $H$ be a subgroup of $G$ such that $A \subseteq H$ and $H / A$ acts on $\lambda$ as Gal $\left(Q_{p}(\lambda) ; Q_{p}(\chi)\right)$. Set $\phi=\lambda^{H}$. Then $m_{Q_{p}}(\phi)=m_{Q_{p}}(\chi)=2$. Suppose $H$ contains no element of order 4. Then a Sylow 2-subgroup of $G$ is elementary abelian and $A$ has a complement $T$ in $H$. Thus $\phi(1)=|T|$ and $\left(\phi,\left(1_{T}\right)^{H}\right)=$ 1 , so $m_{Q_{p}}(\phi)=1$, which is a contradiction. Therefore $H$ contains an element of order 4. Let $x$ be an element of order $q$ in $A$. Since $G$ contains no element of order $4 q$, then $x \notin \mathrm{Z}(H)$. Since $\lambda$ is faithful and $H / A$ acts on $\lambda$ as $\operatorname{Gal}\left(Q_{p}(\lambda) / Q_{p}(\chi)\right)$, then $2 \| Q_{p}\left(\chi, \epsilon_{q}\right): Q_{p} \mid$. Thus, if $k=Q_{P}\left(\epsilon_{q}\right)$, then $m_{k}(\chi)=1$. Therefore $m_{F}(\chi)=1$ for $F=Q\left(\epsilon_{q}\right)$.

Example (1). Define $G=\langle a, b, c, z, x, y, w\rangle$ with the following relations:

$$
\begin{array}{cl}
a^{5}-b^{11}=c^{43}=z^{2}=x^{4}=w^{42}=1, & y^{10}=z, \\
{[x, w]-z, \quad x^{-1} a x=a^{2}, \quad y^{-1} b y=b^{2},} & w^{-1} c u:=c^{3} .
\end{array}
$$

Then $\exp (G)=2^{2} \cdot 3 \cdot 5 \cdot 7 \cdot 11 \cdot 43$. Let $A=\langle a, b, c, z\rangle$ and let $\lambda$ be a faithful linear character of $A$. Then $A \triangleleft G$ and $\chi=\lambda^{G}$ is a rationalvalued irreducible character of $G$. The $p$-local Schur indices of $\chi$ can be calculated by using either the formula of Berman $[1, \S 4]$ or Yamada [4, Chapter 4]. The index $m_{Q_{p}}(\chi)=2$ exactly when $p=5,11,43$, and $\infty$. Furthermore, if $m \in\{4,3,5,7,11,43\}$, there exists $p \in\{5,11,43, \infty\}$ such that $\left|Q_{p}\left(\epsilon_{m}\right): Q_{p}\right|$ is odd. Thus $Q\left(\epsilon_{m}\right)$ fails to split $\chi$ for each such $m$. Hence Brauer's proposition is false.

Example (2). Another example shows that if $\exp (G)$ is replaced by $|G|$, then the proposition is still false. Define $G=\langle a, b, c, z, x, y, w\rangle$ with the following relations:

$$
\begin{gathered}
a^{17}=b^{31}=c^{103}=z^{2}=x^{2}=y^{2}=1, \quad w^{2}=z, \\
{[x, y]=z, \quad x^{-1} a x=a^{-1}, \quad y^{-1} b y=b^{-1}, \quad w^{-1} c w=c^{-1} .}
\end{gathered}
$$

Then $|G|=2^{4} \cdot 17 \cdot 31 \cdot 103$. Set $A=\langle a, b, c, z\rangle, \lambda$ a faithful character of $A$, and $\chi=\lambda^{G}$. Then $\chi$ is real-valued and has local Schur index 2 at 17,31 , and 103. Furthermore, if $m \in\{16,17,31,103\}$, there exists $p \in\{17,31,103\}$ such that $\left|Q_{p}\left(\epsilon_{m}\right): Q_{p}\right|$ is odd. Therefore $Q\left(\epsilon_{m}\right)$ fails to split $\chi$ for each $m$.

The following result shows that this situation cannot happen if $\chi$ is rational-valued.

Proposition. Let $\chi$ be an irreducible character of $G$ such that $Q(\chi)$ is an extension of $Q$ of odd degree. If $|G|=2^{c} n, n$ odd, then $Q\left(\epsilon_{2} c\right)$ splits $\chi$. 
Proof. By the Brauer-Speiser theorem, $m_{Q}(\chi) \leq 2$. By the Brauer-Witt theorem, it suffices to consider $Q(\chi)$-special pairs $(G, \chi)$ where $G / A$ is a 2-group. Since $Q(\chi) / Q$ has odd degree, $G / A$ is isomorphic to a Sylow 2-subgroup of $\mathrm{Gal}(Q(\lambda) / Q)$.

Suppose $m_{Q}(\chi)=2$. Then $\chi$ cannot be linear, so $G \neq A$. Hence 2) $|G: A|$. Let $T$ be a Sylow 2-subgroup of $G$. If $A \cap T=\langle 1\rangle$, then $\chi(1)=$ $|G: A|=|T|$ and $\left(\chi,\left(1_{T}\right)^{G}\right)=1$. In that case, $m_{Q}(\chi)=1$, which is a contradiction. Hence 2||$A \mid$ so 4||$G \mid$ and $c \geq 2$. Thus 2||$Q_{p}\left(\epsilon_{2} c\right): Q_{p} \mid$ for $p=2, \infty$. In particular, $Q_{2}\left(\epsilon_{2} c\right)$ and $Q_{\infty}\left(\epsilon_{2} c\right)$ each split $\chi$.

Let $p$ be an odd prime with $p-1=2^{a} b, b$ odd. Suppose $m_{Q_{p}}(\chi)=2$. Then $p|| A \mid$. Since $\lambda$ is faithful and $G / A$ is isomorphic to a Sylow 2 subgroup of $\mathrm{Gal}(Q(\lambda) / Q), 2^{a}|| G: A \mid$. Therefore, $c \geq a+1$. Hence, 2 ||$Q_{p}\left(\epsilon_{2} c\right): Q_{p} \mid$ so $Q_{p}\left(\epsilon_{2} c\right)$ splits $\chi$.

Therefore $Q\left(\epsilon_{2} c\right)$ splits $\chi$.

\section{REFERENCES}

1. S. D. Berman, Representations of finite groups over an arbitrary field and over rings of integers, Izv. Akad. Nauk SSSR Ser. Mat. 30 (1966), 69-132; English transl., Amer. Math. Soc. Transl. (2) 64 (1967), 147-215. MR 33 \#5747.

2. R. Brauer, Applications of induced characters, Amer. J. Math. 69 (1947), 709-716. MR 9, 268.

3. B. Fein, Realizability of representations in cyclotomic fields, Proc. Amer. Math. Soc. 38 (1973), 40-42. MR $47 \# 3503$.

4. T. Yamada, The Schur subgroup of the Brauer group, Lecture Notes in Math., vol. 397, Springer-Verlag, Berlin and New York, 1974.

DEPARTMENT OF MATHEMATICS, TULANE UNIVERSITY, NEW ORLEANS, LOUISIANA 70118 